05

\title{
Импульсное намагничивание короткозамкнутой катушки из ВТСП
}

\author{
(C) П.А. Бражник, ${ }^{1}$ А.А. Картамышев, ${ }^{1}$ В.С. Коротков, ${ }^{1}$ Е.П. Красноперов, ${ }^{1,2,}$ А.А. Бишаев, ${ }^{3}$ М.В. Козинцева ${ }^{3}$ \\ ${ }^{1}$ Национальный исследовательский центр Курчатовский институт, \\ 123182 Москва, Россия \\ ${ }^{2}$ Московский физико-технический институт, \\ 141700 Долгопрудный, Московская обл., Россия \\ ${ }^{3}$ Московский технологический университет, \\ 119454 Москва, Россия \\ I e-mail: krasnoperov_ep@nrcki.ru
}

Поступило в Редакцию 5 июня 2018 г.

В окончательной редакции 2 августа 2018 г.

Принято к публикации 4 марта 2019 г.

Изучены захваченные магнитные поля и их релаксация при импульсном намагничивании 20-витковой короткозамкнутой катушки из стабилизированной ленты ВТСП-2G при температуре жидкого азота. Наибольшее захваченное поле близко к значению, получаемому при охлаждении в поле (FC process), и слабо изменяется при 5-кратном увеличении амплитуды намагничивающего импульса. Медное покрытие ленты значительно ослабляет эффекты импульсного разогрева. Релаксация тока на начальном участке определена перетеканием тока между сверхпроводящим и нормальным слоями и видом вольт-амперной характеристики. На больших временах $(t>100 \mathrm{~s})$ затухание определено контактным сопротивлением спая.

Ключевые слова: импульсное намагничивание, многовитковая катушка, ВТСП.

DOI: 10.21883/JTF.2019.08.47892.226-18

Ленты из ВТСП-2G имеют широкие перспективы для создания магнитных систем в самых различных областях, таких как медицина (магниторезонансная томография - МРТ), магнитная сепарация, энергетика, магнитное охлаждение и др. Очевидно, что экономическая привлекательность сверхпроводящих магнитных систем зависит от стоимости создания и поддержания магнитного поля. Как показывает практика, наиболее экономичными являются сверхпроводящие системы, работающие в режиме замороженного поля, т. е. в режиме без диссипативной циркуляции тока. Подобный режим достигается в моно доменных ВТСП кольцах $[1,2]$ или в ромбоподобной обмотке, изготовленной из ленты, разрезанной посередине [3]. Провода низкотемпературных сверхпроводников, таких как $\mathrm{NbTi}$ [4] или $\mathrm{MgB}_{2}$ [5] могут быть соединены с очень малым $\left(<10^{-15} \Omega\right)$ контактным сопротивлением. Это в конечном итоге привело к их широкому применению в высокостабильных магнитных системах, таких как МРТ томографы. В ряде технических устройств, например, магнитное охлаждение, сепарация или энергетика, требования к стабильности поля не столь высоки, и небольшой дрейф поля можно компенсировать периодическим подмагничиванием. Подобная процедура является актуальной, если учитывать значительный крип потока в ВТСП материалах. Заметим, что в настоящее время из ленты ВТСП создаются соленоиды с полем до 3 Т, имеющие индуктивность $0.2-0.4 \mathrm{H}$ [6]. Если в таком соленоиде обеспечить контактное сопротивление $R_{C}<10 \mathrm{n} \Omega$, то характерное время затухания поля составит $\tau=L / R_{C} \cong 3 \cdot 10^{7} \mathrm{~s}$, т. е. около года. Подпитывание такой системы с периодич- ностью, скажем, в неделю, может обеспечить стабильность поля лучше 1\%. Таким образом, намагничивание короткозамкнутых сверхпроводящих систем является актуальной задачей.

Намагничивание замкнутой многовитковой сверхпроводящей системы осуществляется двумя способами в зависимости от ее индуктивности. Для систем с большой индуктивностью применяют внешний источник тока и сверхпроводящий ключ [4]. Для мало индуктивных систем перспективен импульсный метод, как наименее энергозатратный.

Импульсное намагничивание дисков и колец из монодоменных ВТСП широко исследуется и достаточно корректно описывается в рамках предположения о степенной вольт-амперной характеристике (BAX) сверхпроводника [7]. При импульсном намагничивании одновиткового кольца могут происходить локальный нагрев узкого сектора кольца и обрыв экранирующего тока [8]. В момент обрыва тока поле в кольце становится равным внешнему полю. Если длительность импульса короче характерного времени остывания перегретой области, то экранирующий ток не возникает, и захваченный поток в кольце (после выключения поля) весьма мал. Более того, поле может иметь обратное направление за счет остаточного намагничения „холодной“ части кольца [8]. В случае, когда длительность спадания намагничивающего поля превышает время остывания канала, то возникшие экранирующие токи обеспечивают максимальный захваченный поток, как в случае FC-процесса [9].

Импульсное намагничивание короткозамкнутой многовитковой катушки (или соленоида) принципиально 


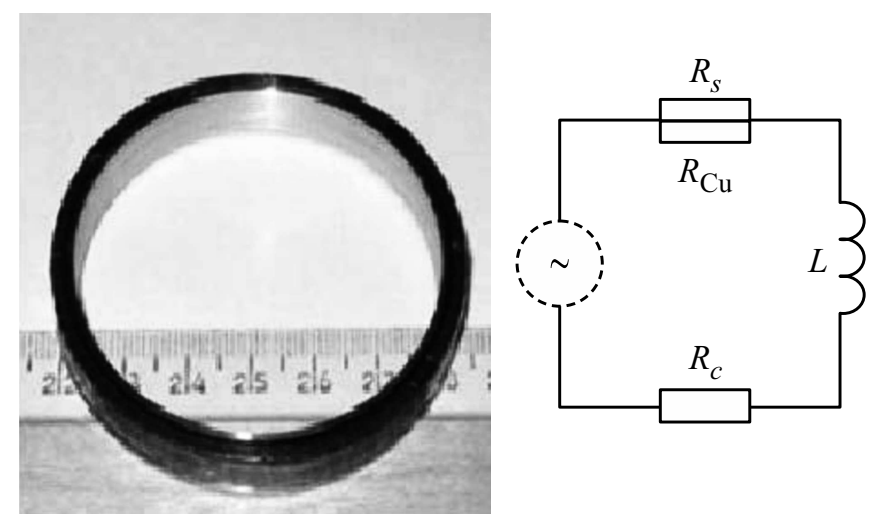

Рис. 1. Фотография двойной спаянной катушки и ее эквивалентная электрическая схема.

отличается от одновиткового кольца (или полого цилиндра). Когда снаружи массивного кольца приложено магнитное поле, экранирующий ток постепенно заполняет сечение сверхпроводника от внешней области к внутренней. В соответствии с моделью критического состояния [10] в области проникновения поля плотность сверхпроводящего тока равна критической, и нулевой в остальной части, где поля нет. В многовитковой катушке экранирующий ток одинаков во всех ее витках, и плотность тока однородна по сечению. В результате разрушение сверхпроводимости наступает тогда, когда экранирующий ток достигает критического значения для данного поля.

В последнее время активно исследуются возможности создания низкоомного (в идеале - сверхпроводящего) соединения лент из ВТСП-2G [11]. Из ленты фирмы „SuperPower“ [12] в работе [13] изготовлены многовитковые катушки, спаянные между собой, и изучено их изотермическое намагничивание в постоянных магнитных полях. Представляло интересным исследовать поведение таких систем в импульсном магнитном поле и влияние медного покрытия на процесс намагничивания.

В настоящей работе изучены токи, возникающие в замкнутой катушке из ленты ВТСП-2G при импульсном намагничивании, и определен характер релаксации тока при температуре жидкого азота.

Двойная катушка из многослойной ленты [12] со спаянными между собой концами изготовлена по методу, изложенному в работе [13]. Фотография катушки диаметром $58 \mathrm{~mm}$, имеющей 20 витков ленты SuperPower, шириной $4 \mathrm{~mm}$ показана на рис. 1. На правой части рисунка изображена эквивалентная электрическая схема, которая отражает структуру ленты [12]. Схема включает: сопротивления сверхпроводящего слоя $R_{s}$ и металлической подложки с медно/серебряными стабилизирующими покрытиями $-R_{\mathrm{Cu}}$. Изображенная схема отличается от обычных параллельно соединенных сопротивлений. Поскольку слои не являются изолированными, а сверхпроводящий слой имеет сугубо нелинейную $\mathrm{BAX}$, из-за перетекания тока их нельзя рассматривать как дискретные элементы. Погонное сопротивление стабилизирующего слоя и подложки при температуре жидкого азота, полученное экстраполяцией из нормального состояния к $T=78 \mathrm{~K}$, составляет $\sim 0.10 \mathrm{~m} \Omega / \mathrm{cm}$. Это значение совпадает с [14], так что полное сопротивление $R_{\mathrm{Cu}}=36 \mathrm{~m} \Omega(l=360 \mathrm{~cm})$. Последовательно в цепи имеется сопротивление двух контактов пайки $-R_{c}$. Рассчитанная индуктивность катушки составляла около $L \cong 38 \mu \mathrm{H}$. Пунктирным кругом обозначена ЭДС $E=\mathrm{Sd} H / d t$, возникающая в кольце во время действия внешнего намагничивающего поля. Осциллограмма поля представляла собой зависимость, близкую к полусинусоиде $H=H_{a} \sin (\pi t / T)$, где $H_{a}-$ амплитуда импульса, $T \cong 6.5 \mathrm{~ms}$ - длительность импульса. Сопротивление сверхпроводящего слоя ленты сугубо нелинейно и определяется ее ВАХ (обычно ВАХ описывается степенной функцией $\left.U=U_{0}\left(I / I_{C}\right)^{N}\right)$.

Ток, возникающий в обмотке катушки при импульсном намагничивании в пределах $10 \mathrm{~ms}$, измерялся с помощью калиброванного пояса Роговского и импульсного осциллографа. Дальнейшая эволюция тока (после окончания импульса) определялась по изменению магнитного поля в центре катушки датчиком Холла, подсоединенного к системе Keihly 6221/2182A. Эта система обеспечивала измерение поля с интервалом $0.1 \mathrm{~s}$ и разрешением по полю $2 \cdot 10^{-5} \mathrm{~T}$.

На рис. 2 изображены эволюции токов в обмотке катушки (левая ось) при разных амплитудах намагничивающего поля. При малых амплитудах $\left(\mu_{0} H_{a} \leq 0.025 \mathrm{~T}\right)$ экранирующие токи меньше критических, и остаточный ток в катушке отсутствует. С ростом $H_{a}$ появляется остаточный ток и соответственно захваченное поле. Заметим, что критический ток исходной ленты, определенный их $\mathrm{BAX}$ на уровне $0.1 \mu \mathrm{V} / \mathrm{cm}$, составлял $100 \mathrm{~A}$ [12].

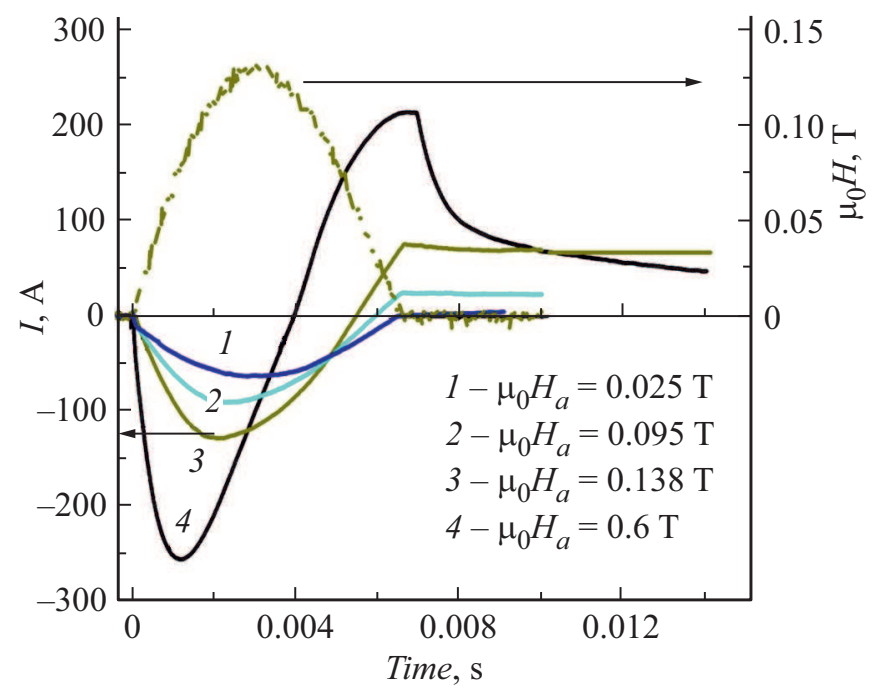

Рис. 2. Токи в кольце (левая ось) при намагничивании амплитудами $\mu_{0} H_{a}$ сверху вниз: $0.025,0.095,0.138$ и $0.6 \mathrm{~T}$. Пунктир - импульс с $\mu_{0} H_{a}=0.138 \mathrm{~T}$ (правая ось). 


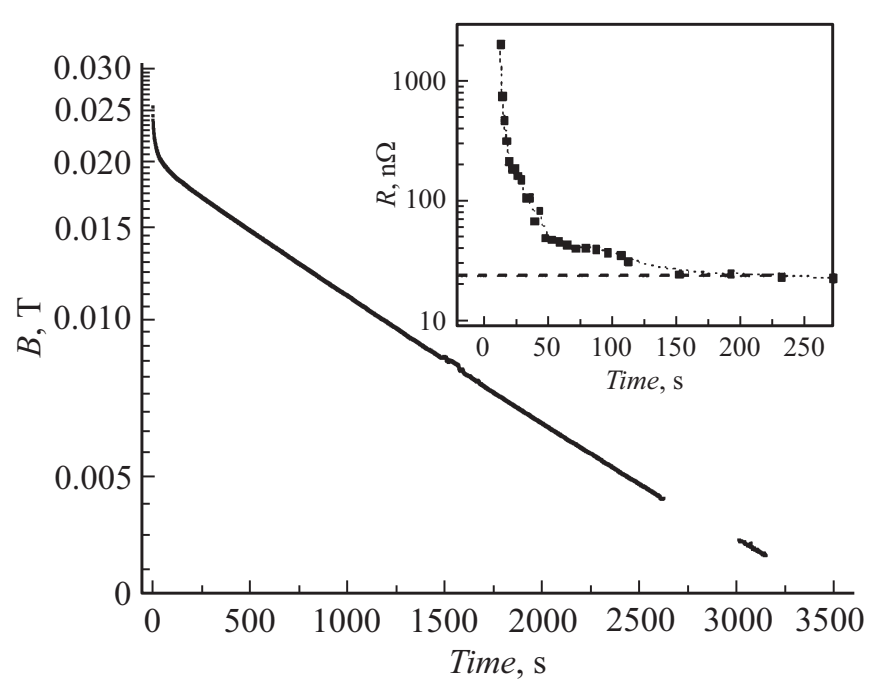

Рис. 3. Релаксация поля в многовитковой короткозамкнутой катушке. Поле измерялось в Т.

В результате спаивания концов катушки критический ток, по оценке [13], снизился до 54.3 А. Это могло быть как следствием влияния магнитного поля катушки на ток, так и деградацией сверхпроводимости на длине пайки.

После окончания внешнего импульса оставшееся в кольце поле снижается со временем из-за релаксации тока. На рис. 3 приведена зависимость от времени $\ln B(t)$. В логарифмическом масштабе хорошо различимы два участка: быстрая релаксация (при $t<100 \mathrm{~s}$ ) и медленная релаксация (при $t>200 \mathrm{~s}$ ), которая идеально описывается экспоненциальным законом $B=B_{0} \exp \left(-t / \tau_{c}\right)$, где $\tau_{c}=1601 \pm 1 \mathrm{~s}$. Релаксацию тока в соответствии с эквивалентной схемой (рис. 1) можно описать известным уравнением

$$
L d i / d t+\left(R^{*}+R_{c}\right) i=0 .
$$

Здесь $R^{*}$ - сопротивление параллельных слоев меди (с подложкой) $R_{\mathrm{Cu}}$ и сверхпроводящего слоя $R_{s} ; R_{c}-$ сопротивление паянных контактов.

Большое время $\tau_{c}$ характеризует диссипацию энергии на паяных контактах, общее сопротивление которых составляет $R_{c}=L / \tau_{c} \approx 24 \mathrm{n} \Omega$. Учитывая, что контактов два и площадь пайки каждого $4 \mathrm{~cm}^{2}$, можно заключить, что примененная технология пайки [13] обеспечивает соединение лент с сопротивлением $\sim 48 \mathrm{n} \Omega \cdot \mathrm{cm}^{2}$.

Начальный участок релаксационной кривой $(t<100 \mathrm{~s})$ сложен для интерпретации, поскольку характеризует перетекание тока между сверхпроводящим слоем с нелинейной $\mathrm{BAX}$ и стабилизирующим медным покрытием. При этом сопротивления подложки и покрытия можно считать постоянными, а сопротивление сверхпроводящего слоя изменяется в соответствии с ВАХ. Если ток течет только в медном покрытии с $R_{\mathrm{Cu}}=36 \mathrm{~m} \Omega$, то время его затухания оказывается очень коротким
$\tau_{c}=L / R_{\mathrm{Cu}} \cong 1 \mathrm{~ms}$, и в рассматриваемом масштабе времени (десятки секунд) релаксацию тока в меди можно не учитывать. Если начальный участок релаксационной кривой на рис. 3 описывать степенной $\mathrm{BAX}$, то он будет соответствовать изменяющемуся индексу $N$ от 18 до 22. Феноменологически релаксацию тока на начальном участке удобно характеризовать эффективным сопротивлением $R^{*}(t)$, полагая, что на коротком интервале времени закон релаксации остается экспоненциальным, но с изменяющимся временем релаксации $\tau(t)=L / R^{*}(t)$. Дифференцированием кривой на рис. 3 получена зависимость $R^{*}(t)$, которая изображена в рамке на рисунке. Легко видеть, что сопротивление снижается по крайне мере на 2 порядка, и приближается к сопротивлению спаев $R_{c}=24 \mathrm{n} \Omega$.

Импульсное намагничивание массивного сверхпроводника обычно протекает в адиабатических условиях, поскольку время теплообмена с термостатом значительно превышает длительность импульса. Вследствие этого величина захваченного поля в зависимости от амплитуды имеет узкий максимум [15]. В области температур $T=78 \mathrm{~K}$ захваченные поля при импульсном намагничивании примерно такие же, как в случае изотермического намагничивания.

С понижением температуры и ростом критического тока проявляется отрицательная сторона импульсного нагрева. Снижение теплоемкости и необходимое увеличение амплитуды $H_{a}$ вызывают рост температуры в десятки градусов, что в $2-3$ раза снижает импульсное $B_{t r}$ по сравнению с изотермическим FC-процессом [16].

Нами изучено влияние амплитуды намагничивающего импульса на величину захваченного поля. На рис. 4 представлены эволюции захваченных полей для различных амплитуд намагничивания. Захваченные поля

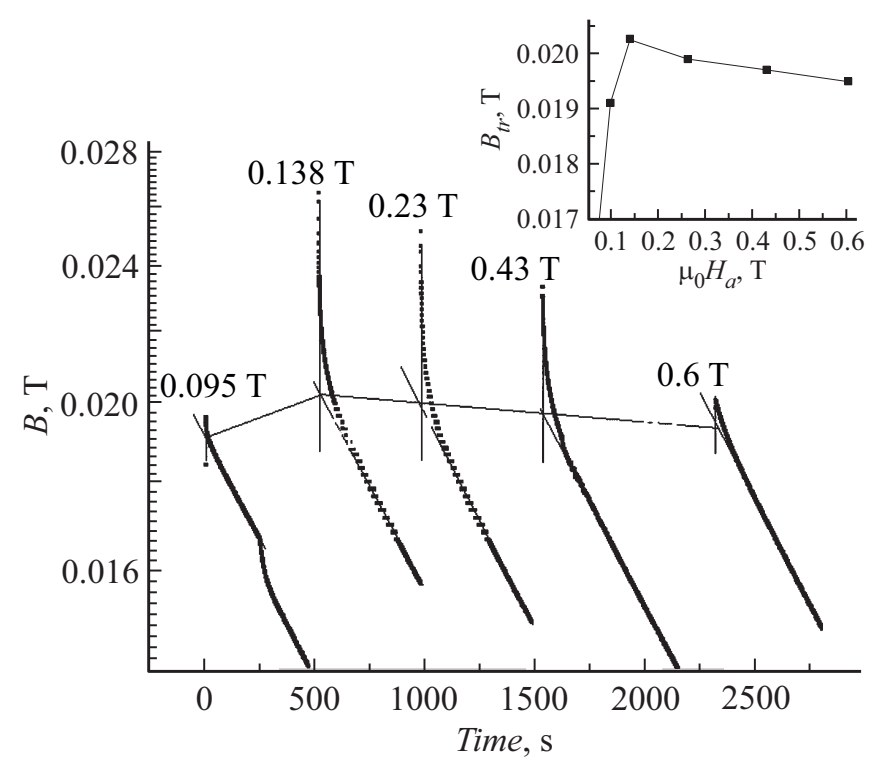

Рис. 4. Релаксация захваченных полей при разных амплитудах намагничивающих импульсов. Слева направо $\mu_{0} H_{a}=0.095$, $0.138,0.23,0.43$ и $0.6 \mathrm{~T}$. 
(ось ординат) отложены в логарифмическом масштабе, поле $B$ измерялось в Т. Параллельные с одинаковым наклоном линии, как показано выше, характеризуют релаксацию тока на контактном сопротивлении, а их пересечение с вертикальными линиями дают начальные значения остаточного сверхпроводящего тока, который обеспечивает захваченное поле $B_{t r}$. Во вставке на рис. 4 показана зависимость $B_{t r}\left(H_{A}\right)$. При малых амплитудах $\left(\mu_{0} H_{a}=0.025 \mathrm{~T}\right)$ циркулирующий по обмотке ток ниже критического и он полностью экранирует внешнее поле $\left(B_{t r}=0\right)$. С ростом $H_{a}$ появляется захваченное поле. Затем поле достигает максимума, и в последующем $B_{t r}$ снижается на 6\% при 5-кратном увеличении амплитуды. Максимально захваченное поле составляет около $B_{t r}=0.022 \mathrm{~T}$ при $\mu_{0} H_{a}=0.138 \mathrm{~T}$. Если для этого поля рассчитать циркулирующий по катушке ток, то он оказывается близким к значению $I=54.3$ А, полученному при медленном охлаждении в поле [13].

Снижение $B_{t r}$ объясняется нагревом сверхпроводника и соответственно падением критического тока. Благодаря плотной намотке ленты и ее изоляции, а также малой длительности импульса $(6.5 \mathrm{~ms})$, можно считать, что нагрев происходит в адиабатических условиях. Верхняя примитивная оценка температуры нагрева может быть сделана в предположении, что весь ток течет по меди. В этом случае выделившееся тепло можно рассчитать из осциллограммы тока в ленте (рис. 2). В силу малой длительности импульса по сравнению со временем охлаждения, можно оценить нагрев ленты, полагая, что ток перетекает в медное покрытие, когда его величина превышает критическое значение $I_{c}$. Т. е. выделившееся тепло представить как $Q=\int I^{2} R_{\mathrm{Cu}} d t$ в пределах времени, когда общий ток $I>I_{c}$. Затем, используя объемную теплоемкость $C_{v}=1.8 \mathrm{~J} /\left(\mathrm{cm}^{3} \cdot \mathrm{K}\right)[14]$, найдем повышение температуры $\Delta T$ и снижение критического тока. Для наибольшей амплитуды $\left(\mu_{0} H_{a}=0.6 \mathrm{~T}\right)$ выделившееся тепло увеличит температуру на $5 \mathrm{~K}$. Соответственно падение критического тока и $B_{t r}$ составит $40 \%$. Если же только половина общего тока течет по меди, то падение $B_{t r}$ составит $10 \%$, что близко к наблюдаемому значению.

Слабый нагрев сверхпроводника при значительном изменении амплитуды импульса объясняется стабилизирующим действием медного покрытия. Точно так же, как при стационарной стабилизации, предложенной в работе [17] для обычных сверхпроводников, ток перетекает в медное покрытие, когда он превышает критическое значение. В нашем случае толщина медного покрытия на 2 порядка больше сверхпроводящего слоя. С учетом малой длительности импульса и высокой проводимости меди тепловыделение оказывается небольшим, и критический ток снижается незначительно.

Оптимизация параметров импульса (амплитуда и длительность) для достижения наибольшего захваченного поля в катушке с заданными свойствами, такими как толщина медного покрытия, критический ток и индук- тивность, требует дополнительных расчетов и экспериментальных исследований.

\section{Заключение}

Определены захваченные магнитные поля и их релаксация при импульсном намагничивании короткозамкнутой (спаянной) многовитковой катушки из стабилизированной ленты ВТСП-2G при $T=78 \mathrm{~K}$. Сопротивление контакта, определенное из скорости затухания поля, составило $\sim 48 \mathrm{n} \Omega$ на площади спая $1 \mathrm{~cm}^{2}$. Когда экранирующий ток в катушке превышает критический, он из сверхпроводящего слоя перетекает в медное покрытие. Толстый слой меди на ленте снижает импульсный нагрев сверхпроводника. В результате 5-кратное увеличение амплитуды намагничивающих импульсов снижает критический ток менее чем на 10\%. Наблюдаемый эффект может оказаться полезным при низких температурах, где, как говорилось выше, из-за снижения теплоемкости импульсно захваченные поля в $2-3$ раза ниже, чем при изотермическом намагничивании. Благодаря увеличению проводимости меди с понижением температуры можно ожидать ослабление импульсного нагрева, что позволит увеличить амплитуду импульса и соответственно захваченные поля в ленточных многовитковых замкнутых катушках с медным стабилизирующим покрытием.

\section{Благодарности}

Авторы благодарны А.А. Шикову за измерения сопротивления ленты.

\section{Финансирование работы}

Работа выполнена при финансовой поддержке Министерства образования и науки Российской Федерации в рамках соглашения № 14.604.21.0197 о предоставлении субсидии (уникальный идентификатор прикладных научных исследований RFMEFI60417X0197).

\section{Конфликт интересов}

Авторы заявляют, что у них нет конфликта интересов

\section{Список литературы}

[1] Masaru Tomita, Yusuke Fukumoto, Kenji Suzuki, Atsushi Ishihara, Miryala Muralidhar // J. Appl. Phys. 2011. Vol. 109. P. 023912.

[2] Korotkov V.S., Krasnoperov E.P., Kartamyshev A.A. // J. Supercond. Nov Magn. 2014. Vol. 27. P. 1845.

[3] Sheng J., Zhang M., Wang Y., Li X., Pateland J., Yuan W. // Supercond. Sci. Technol. 2017. Vol. 30. P. 094002.

[4] Алексеевский Н.Е., Красноперов Е.П. // ДАН. 1970. № 197. C. 1325.

[5] Ling J., Voccio J., Kim Y., Hahn S., Bascuñán J., Park D.K., Iwasa Y. // IEEE. Trans. Appl. Supercond. 2013. Vol. 23. N 3 P. 4601705 . 
[6] Maeda H., Yanagisawa Y. // IEEE. Trans. Appl. Supercond. 2014. Vol. 24. N 3. P. 1-12.

[7] Ainslie M.D., Fujishiro H., Ujiie T. et al. // Supercond. Sci. Technol. 2014. Vol. 27. P. 065008.

[8] Korotkov V.S., Krasnoperov E.P., Kartamyshev A.A. // J. Supercond. Nov Magn. 2016. Vol. 29. P. 1893-1896.

[9] Красноперов Е.П., Коротков В.С., Картамышев А.А. // Письма в ЖТФ. 2017. Т. 43. Вып. 19. С. 28. [Krasnoperov E.P., Korotkov V.S., Kartamyshev A.A. // Tech. Phys. Lett. 2017. Vol. 43. N 10. P. 882-884.]

[10] Swartz P.S., Bean C.P. // J. Appl. Phys. 1968. Vol. 39. P. 4991.

[11] Ohki K., Nagaishi T., Kato T. et al. // Supercond. Sci. Technol. 2017. Vol. 30. P. 115017.

[12] Электронный ресурс. Режим доступа: $\mathrm{http} / / / \mathrm{www}$. superpower-inc.com/

[13] Козинцева М.В., Бишаев А.М., Буи А.А., Гавриков М.Б., Каменцев К.Е., Нижельский Н.А., Савельев В.В., Сигов А.С. // ЖТФ. 2017. Т. 87. Вып. 6. С. 875.

[14] Pelegrin J., Martinez E., Angurel L.A., Yi-Yuan Xie, Selvamanickam V. // IEEE. Trans. Appl. Supercond. 2011. Vol. 21. N 3. P. 3017.

[15] Kartamyshev A.A., Krasnoperov E.P., Kuroedov Yu.D., Nizhelskiy N.A., Poluschenko O.L. // Physica C. 2009. Vol. 469. P. 805-809.

[16] Yanagi Y., Itoh Y., Yoshikawa M., Oka T. // Sci. Technol. 2005. Vol. 18. P. 839-849.

[17] Stekly Z.J.J., Zar J.L. // IEEE Trans. Nucl. Sci. 1965. Vol. 2. P. 367. 